\title{
lon engineering of embedded nanostructures: From spherical to facetted nanoparticles
}

\author{
G. Rizza, ${ }^{1, a)}$ E. A. Dawi, ${ }^{2}$ A. M. Vredenberg, ${ }^{2}$ and I. Monnet $^{3}$ \\ ${ }^{1}$ Ecole Polytechnique, Laboratoire des Solides Irradiés, CEA-IRAMIS-CNRS, \\ 91128 Palaiseau Cedex, France \\ ${ }^{2}$ Debye Institute for Nanomaterials, Nanophotonics Section, Utrecht University, P.O. Box 80.000, \\ 3508 TA Utrecht, The Netherlands \\ ${ }^{3}$ CIMAP-ENSICAEN-CEA-CNRS-University of Caen, Bd H. Becquerel, BP 5133, 14070, \\ Caen Cedex 5, France
}

(Received 5 June 2009; accepted 3 July 2009; published online 28 July 2009)

\begin{abstract}
We show that the high-energy ion irradiation of embedded metallic spherical nanoparticles (NPs) is not limited to their transformation into prolate nanorods or nanowires. Depending on their pristine size, the three following morphologies can be obtained: (i) nanorods, (ii) facettedlike, and (iii) almost spherical nanostructures. Planar silica films containing nearly monodisperse gold NPs $(8-100 \mathrm{~nm})$ were irradiated with swift heavy ions $(5 \mathrm{GeV} \mathrm{Pb})$ at room temperature for fluences up to $5 \times 10^{13} \mathrm{~cm}^{-2}$. The experimental results are accounted for by considering a liquid-solid transformation of the premelted NP surface driven by the in-plane stress within the ion-deformed host matrix. This work demonstrates the interest of using ion-engineering techniques to shape embedded nanostructures into nonconventional configurations. (C) 2009 American Institute of Physics. [DOI: 10.1063/1.3186030]
\end{abstract}

Amorphous materials subjected to high-energy ion irradiation show irreversible anisotropic plastic flow at temperatures far below the glass transition temperature. ${ }^{1}$ They shrink in the direction of the ion beam and expand in the direction perpendicular to it. On the other hand, for crystalline materials direct irradiation-induced deformation has never been observed. To overcome this limitation, a new strategy has been recently adopted to shape metallic nanoparticles (NPs). Deformation can be induced indirectly by embedding the NPs into an ion-deformable amorphous host matrix. ${ }^{2-6}$ With this technique, spherical NPs deform into prolate nanorods and nanowires, with an aspect ratio that can be tuned by varying the irradiation conditions (ion type, energy and fluence). In this work, we show that the ion-shaping mechanism is not only limited to the transformation of spherical NPs into prolate nanorods/nanowires, but that, depending on the NP size and irradiation conditions, a different class of ionshaped NPs can be obtained, namely, embedded NPs with a facettedlike morphology. This work widens the potentialities of the ion-engineering technique to shape embedded nanostructures into nonconventional configurations, allowing, simultaneously, to tune the optical features of the corresponding composite glass. ${ }^{7,8}$

Monodisperse spherical gold NPs, with average diameters of $8,15,50,80$, and $100 \mathrm{~nm}$ (size dispersion 10\%), were confined within a $350 \mathrm{~nm}$ thick silica film deposited onto a silicon substrate. All the NPs are in a single plane 150 $\mathrm{nm}$ below the sample surface, such that the energy deposited is the same for all the NPs. For more details about the sample preparation we refer the reader to the literature. ${ }^{6,9}$ The experiments were carried out with the aid of the GANIL facilities in Caen (France). High energy (HE) was used to obtain 5 $\mathrm{GeV} \mathrm{Pb}$ ions. Samples were irradiated at room temperature $(300 \mathrm{~K})$ and at normal incidence for fluences ranging from

${ }^{\text {a)} E l e c t r o n i c ~ m a i l: ~ g i a n c a r l o . r i z z a @ p o l y t e c h n i q u e . e d u . ~}$
$1 \times 10^{13}$ up to $5 \times 10^{13} \mathrm{~cm}^{-2}$. In order to avoid any macroscopic heating, the ion flux was limited to 3 $\times 10^{8}$ ions $\mathrm{cm}^{-2} \mathrm{~s}^{-1}$. The electronic stopping power of the $\mathrm{Pb}$ ions in both $\mathrm{SiO}_{2} \quad\left(17 \mathrm{keV} \mathrm{nm}^{-1}\right)$ and gold $\left(76 \mathrm{keV} \mathrm{nm}^{-1}\right.$ ) were calculated with the SRIM 2008 code. ${ }^{10}$ After preparation in cross-section geometry [(cross-sectional transmission electron microscopy (TEM)], the irradiated samples were analyzed using CM30 and Tecnai F20 microscopes. TEM micrographs were processed with a slow-scan camera and analyzed with the Digital micrograph program. The average NP sizes and their dispersion have been determined by considering the corresponding size distribution profiles. These were obtained by analyzing at least 100 particles for each sample.

Figures 1(a)-1(e) show TEM micrographs of Au NPs of different sizes $(8-100 \mathrm{~nm})$ after irradiation with $5 \mathrm{GeV} \mathrm{Pb}$ ions at a fluence of $5 \times 10^{13} \mathrm{~cm}^{-2}$. From the inspection of these figures, we can infer that there are two populations of NPs: (i) NPs larger than $80 \mathrm{~nm}$ conserve their spherical shape after SHI irradiation. (ii) smaller NPs become elongated along the beam direction. Usually, the as-prepared NPs can be characterized by estimating their diameter, $D_{0}$, whereas ion-deformed NPs can be described by considering their major and minor axes, i.e., $D_{\max }$ and $D_{\min }$. Figure 2 shows the evolution of the normalized major axis $\left(D_{\max } / D_{0}\right)$ as a function of the initial NP size $\left(D_{0}\right)$ for two irradiation fluences: $1 \times 10^{13} \mathrm{~cm}^{-2}$ (open circles) and $5 \times 10^{13} \mathrm{~cm}^{-2}$ (full circles). In both cases, we observe a linear decrease of
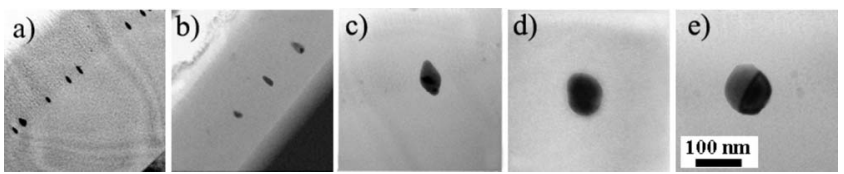

FIG. 1. Bright-field TEM micrograph of gold NPs irradiated at a fluence of $5 \times 10^{13} \mathrm{~cm}^{-2}$. (a) $8 \mathrm{~nm}$, (b) $15 \mathrm{~nm}$, (c) $50 \mathrm{~nm}$, (d) $80 \mathrm{~nm}$, and (e) $100 \mathrm{~nm}$. 


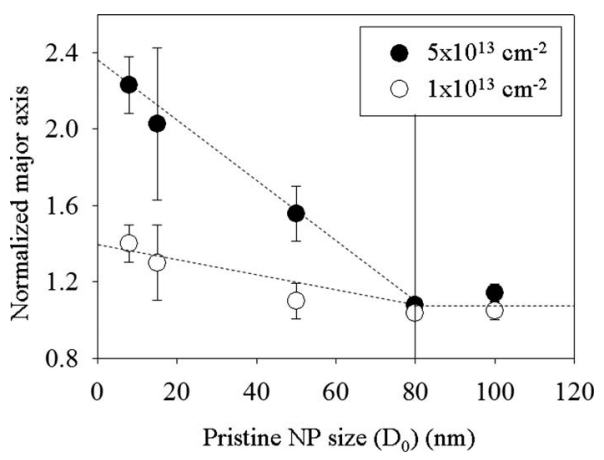

FIG. 2. Evolution of the normalized major axes $\left(D_{\max }\right)$ as a function of the pristine NP size for irradiation fluences of $1 \times 10^{13} \mathrm{~cm}^{-2}$ (open circles) and $5 \times 10^{13} \mathrm{~cm}^{-2}$ (full circles).

the ratio with the size of the pristine NP. In particular, for NPs larger than $80 \mathrm{~nm}$, the deformation is almost completely suppressed. This confirms previous observations, see, e.g., Ref. 11, indicating that the process is somehow related to the initial volume of the NPs, i.e., the larger the NP the larger its inertia against ion deformation.

Focusing our attention to the ion-deformed NPs only $\left(D_{0}<80 \mathrm{~nm}\right)$, we observe that we can discern two subcategories: (i) 8 and $15 \mathrm{~nm}$ NPs transform into the usual prolate rodlike shape, whereas (ii) the spherical NPs whose initial diameter is $50 \mathrm{~nm}$ evolve toward a facetted shaped, Figs. $3(\mathrm{a})$ and $3(\mathrm{~b}){ }^{12}$ It is worth mentioning that the latter shape (50 nm NPs) most likely does not correspond to the steadystate morphology for the irradiated NPs, i.e., the nanorod/ nanowire shape. It is therefore all the more noteworthy that a careful control of (i) the electronic stopping power, (ii) the pristine NP size, and (iii) the irradiation fluence, permits to ion-shape NPs into a facetted configurations within an amorphous matrix. Moreover, although all the irradiated NPs present similar morphologies, they are not always made of a single grain, see, e.g., Fig. 3(b). The presence of grain boundaries in irradiated NPs reveals that the transformation takes place independently for each grain, i.e., to be effective the process does not require that the whole volume of the NP
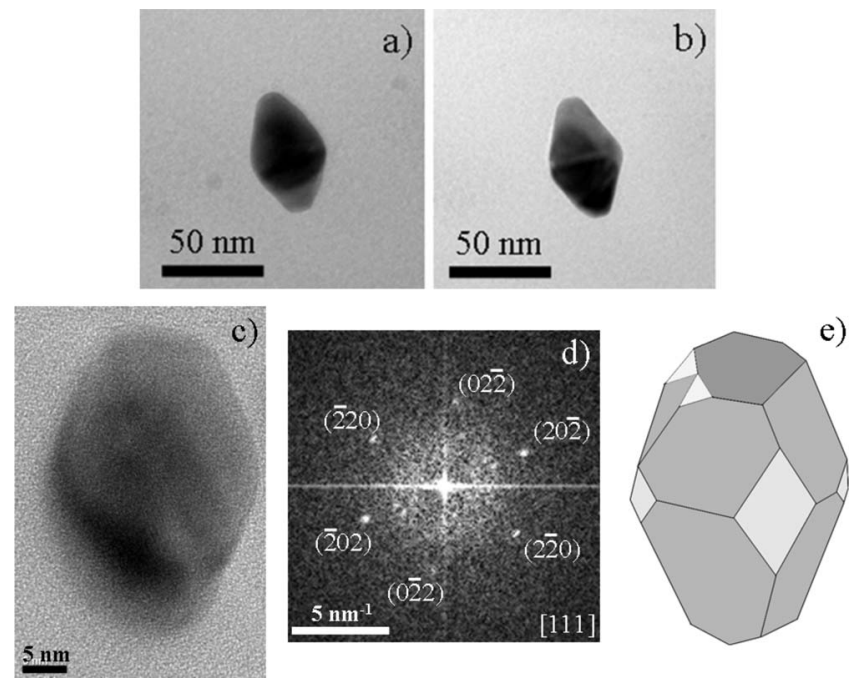

FIG. 3. [(a) and (b)] TEM micrographs of two $50 \mathrm{~nm}$ NPs irradiated at a fluence of $5 \times 10^{13} \mathrm{~cm}^{-2}$. (a) Single crystal NP, (b) NP formed of two grains, (c) HRTEM micrograph, (d) diffraction pattern of an ion-shaped $50 \mathrm{~nm} \mathrm{NP}$, and (e) 3D reconstruction of from the HRTEM image. contributes simultaneously to the transformation. High resolution TEM (HRTEM) of a single crystal irradiated NP and the corresponding diffractrogram are shown in Figs. 3(c) and $3(d)$. The latter shows that the facettes have the $\{110\}$ orientation. However, this does not correspond to one of the most stable forms, i.e., the truncated octahedron, where the crystal surface is dominated by $\{111\}$ and $\{100\}$ forms. ${ }^{12,13}$ Figure 3(e) shows the three-dimensional (3D) reconstruction of the NP shape obtained from the HRTEM micrograph. Finally, a similar transformation has also been observed for $40 \mathrm{~nm}$ $\mathrm{Au}-\mathrm{Ag}$ alloy NP irradiated with $90 \mathrm{MeV} \mathrm{Xe}$ ions,${ }^{14}$ e.g., for a stopping power on the low energy side of the Bragg peak. Thus, this transformation represents a general behavior of the irradiated NPs, where the key parameter is the size rather than the stopping power (within a certain extend).

Although by now several observations of the ionshaping process have been reported in the literature, the underlying mechanism driving it has not been completely elucidated yet, even though a few theoretical studies have been attempted. ${ }^{2,11,15,16}$ In the most reasonable mechanism proposed hitherto, one assumes that the effects of stress and the thermal spike combine to induce the elongation of the metallic NPs. ${ }^{11,16,17}$ Elongation will only occur when the temperatures of both the metallic NP and the dielectric $\mathrm{SiO}_{2}$ matrix exceed their respective individual melting temperatures within the ion track, i.e., the elongation of embedded NPs necessitates the flow of metallic species into the liquid silica track. However, numerical simulations indicate that the energy deposited into the electronic subsystems can only melt metallic NPs whose diameters are smaller than 20 $\mathrm{nm}^{2,11}$ Thus, $50 \mathrm{~nm}$ NPs cannot melt and the previous mechanism for deformation cannot be adopted.

Two different pathways can be invoked to account for the morphological transformation of the NPs under irradiation: the solid-solid and the liquid-solid transformation. The first mechanism, is driven by the diffusion of defects. These become mobile at some temperature and tend to migrate to the interface, where they remain in motion. Although at the nanoscale the diffusion coefficient can be larger than that at the macroscopic scale,${ }^{18,19}$ the very rapid relaxation time of the lattice temperature for the embedded Au NPs, e.g., $10^{-10} \mathrm{s,}{ }^{11,20}$ associated with the fact that the transformation is completed within a fluence of $5 \times 10^{13} \mathrm{~cm}^{-2}$, render this mechanism quite improbable. The liquid-solid transformation refers to surface premelting. In fact, often the melting transition is preceded by premelting phenomena, where a liquid layer is first formed on the solid surface and continues to thicken with temperature increase until the solid core melts. This mechanism was used to explain shape transformations of $\mathrm{Au} \mathrm{NPs},{ }^{21}$ at temperatures lower than the particle melting temperature. Recently, Ruan et al., ${ }^{22}$ have experimentally observed the reversible surface premelting of $\mathrm{Au}$ NPs $(2-20 \mathrm{~nm})$ under femtosecond laser irradiation. Experimental observations of surface premelting in $61.5 \mathrm{~nm} \mathrm{Au}$ NPs were reported by Plech et al. ${ }^{23}$

If the existing models state that NPs larger than $20 \mathrm{~nm}$ cannot melt, this does not imply that they cannot be heated up. The corresponding temperature increase will depend on the NP size, i.e., the larger the NP the less it will heat up. However, the critical melting size of the NPs, $20 \mathrm{~nm}$, must be considered with caution. The reason is that the models/ simulations contain very crude approximations for the potential barrier at the metal/dieletric interface (Kapitza resis- 
tance). This barrier, which depends on the band structure of the two interfacial materials, controls the heat transport driven by electrons and phonons. For the $\mathrm{Au} / \mathrm{SiO}_{2}$, this interface barrier is about $4 \mathrm{eV}^{24}$ Such a high barrier may reduce the heat spreading. It will result in an increase of both the temperature peak within the metal NP, i.e., the melting of larger NPs, as well as the relaxation time of the heated region, i.e., enhancement of the diffusion time.

For NPs in the range of $40-60 \mathrm{~nm}$, it is likely that the temperature elevation is not too far from the melting temperature such that their surfaces may transform into liquid layers. For Au NPs in the range 10-60 nm, Sambles ${ }^{25}$ estimates a liquid layer thickness as high as $2.2 \mathrm{~nm}$. If we take this value for our NPs, this corresponds to about $25 \%$ for the volume of the NP that is in a liquid state. Thus, the facetting process is probably associated with the epitaxial reconstruction from the core of the NP which remains in the solid state. For larger NPs, with diameter between 80 and $100 \mathrm{~nm}$, the temperature increase either is not sufficient to activate the surface premelting or the premelted region is too thin, such that the deformation of the NPs becomes strongly reduced or negligible.

Finally, our NPs are confined within an ion-deformable matrix. In this case, during the irradiation, a compressive in-plane stress is built-up within the matrix. ${ }^{16}$ The irradiation-induced stress, acting on the NPs, may thus modify their surface energies, $\gamma_{(h k l)}$, and surface tensions, $\sigma_{(h k l)}$, and thus alter the energetic configurations. As the morphological change must occur during the surface premelting this can be seen as a liquid-solid transformation process mediated by the in-plane stress generated within the silica matrix by the impinging ions.

G.R. wishes to thank M. Toulemonde, B. Gervais and G. Coddens for the many fruitful discussions and suggestions and Dominique Delille for the assistance with the Tecnai microscope. This work was supported by the METSA network.
${ }^{1}$ S. Klaumünzer and G. Schumacher, Phys. Rev. Lett. 51, 1987 (1983).

${ }^{2}$ C. D'Orléans, J. P. Stoquert, C. Estournès, C. Cerruti, J. J. Grob, J. L. Guille, F. Haas, D. Muller, and M. Richard-Plouet, Phys. Rev. B 67, 220101(R) (2003).

${ }^{3}$ A. Oliver, J. A. Reyes-Esqueda, J. C. Cheang-Wong, C. E. RománVelázquez, A. Crespo-Sosa, L. Rodríguez-Fernández, J. A. Seman, and C. Noguez, Phys. Rev. B 74, 245425 (2006).

${ }^{4}$ Y. K. Mishra, F. Singh, D. K. Avasthi, J. C. Pivin, D. Malinovska, and E. Pippel, Appl. Phys. Lett. 91, 063103 (2007).

${ }^{5}$ R. Giulian, P. Kluth, L. L. Araujo, D. J. Sprouster, A. P. Byrne, D. J. Cookson, and M. C. Ridgway, Phys. Rev. B 78, 125413 (2008).

${ }^{6}$ E. A. Dawi, G. Rizza, M. P. Mink, A. M. Vredenberg, and F. H. P. M. Habraken, J. Appl. Phys. 105, 074305 (2009).

${ }^{7}$ U. Kreibig and M. Vollmer, Optical Properties of Metal Clusters (Springer, Berlin, 1995).

${ }^{8}$ S. Link and M. A. El-Sayed, J. Phys. Chem. B 103, 4212 (1999).

${ }^{9}$ G. Rizza, H. Cheverry, T. Gacoin, A. Lamasson, and S. Henry, J. Appl. Phys. 101, 014321 (2007).

${ }^{10}$ J. F. Ziegler, J. P. Biersack, and U. Littmark, The Stopping Ranges and Ranges of Ions in Solids (Pergamon Press, New York, 1985); www.srim.org.

${ }^{11}$ K. Awazu, X. Wang, M. Fujimaki, J. Tominaga, H. Aiba, Y. Ohki, and T. Komatsubara, Phys. Rev. B 78, 054102 (2008).

${ }^{12}$ G. Z. Wulff, Z. Kristallogr. Mineral. 34, 449 (1901).

${ }^{13}$ A. S. Barnard, X. M. Lin, and L. A. Curtiss, J. Phys. Chem. B 109, 24465 (2005).

${ }^{14}$ G. Rizza, E. A. Dawi, A. M. Vredenberg, and I. Monnet (unpublished).

${ }^{15}$ S. Roorda, T. van Dillen, A. Polman, C. Graf, A. M. Vredenberg, A. van Blaaderen, and B. Kooi, Adv. Mater. 16, 235 (2004).

${ }^{16}$ S. Klaumünzer, Nucl. Instrum. Methods Phys. Res. B 244, 1 (2006).

${ }^{17}$ P. Kluth, R. Giulian, D. J. Sprouster, C. S. Schnohr, A. P. Byrne, D. J. Cookson, and M. C. Ridgway, Appl. Phys. Lett. 94, 113107 (2009).

${ }^{18}$ Q. Jiang, S. H. Zhang, and J. C. Li, Solid State Commun. 130, 581 (2004).

${ }^{19}$ G. Guisbiers and L. Buchaillot, Nanotechnology 19, 435701 (2008).

${ }^{20}$ Z. G. Wang, C. Dufour, E. Paumier, and M. Toulemonde, J. Phys.: Condens. Matter 6, 6733 (1994); 7, 2525 (1995).

${ }^{21}$ S. Inasawa, M. Sugiyama, and Y. Yamaguchi, J. Phys. Chem. B 109, 3104 (2005).

${ }^{22}$ C.-Y. Ruan, Y. Murooka, R. K. Raman, and R. A. Murdicket, Nano Lett. 7, 1290 (2007)

${ }^{23}$ A. Plech, R. Cerna, V. Kotaidis, F. Hudert, A. Bartels, and T. Dekorsyet, Nano Lett. 7, 1026 (2007).

${ }^{24}$ J. Robertson, J. Vac. Sci. Technol. B 18, 1785 (2000).

${ }^{25}$ J. R. Sambles, Proc. R. Soc. London, Ser. A 324, 339 (1971). 